\author{
Pablo Galindo-Moreno \\ Ildefonso Moreno-Riestra \\ Gustavo Ávila \\ Juan Emilio \\ Fernández-Barbero \\ Francisco Mesa \\ Mariano Aguilar \\ Hom-Lay Wang \\ Francisco O'Valle
}

\section{Histomorphometric comparison of maxillary pristine bone and composite bone graft biopsies obtained after sinus augmentation}

Authors' Affiliations:

Pablo Galindo-Moreno, Oral Surgery and Implant

Dentistry Department, School of Dentistry,

University of Granada, Granada, Spain

Ildefonso Moreno-Riestra, Gustavo Ávila,

Hom-Lay Wang, Department of Periodontics and

Oral Medicine, School of Dentistry, University of

Michigan, Ann Arbor (MI), USA

Juan Emilio Fernández-Barbero, Human Anatomy and Embryology Department, School of Medicine, University of Granada, Granada, Spain

Francisco Mesa, Periodontology Department,

School of Dentistry, University of Granada, Spain Mariano Aguilar, Francisco O'Valle, Pathology

Department, School of Medicine, University of

Granada, Granada, Spain

\section{Correspondence to:}

Dr Pablo Galindo-Moreno

$\mathrm{C} /$ Recogidas

$395^{\circ} \mathrm{Izq}$

I8005 Granada

Spain

Tel.: + 34958520658

Fax: + 34958520658

e-mail: pgalindo@ugr.es

Key words: anorganic bovine bone, autologous bone, bone remodeling, dental implants, sinus grafting

\begin{abstract}
Introduction: Sinus grafting is a technique oriented to facilitate implant placement in posterior atrophic maxillae. Several modifications of the original technique and a wide variety of materials have been proposed; most of them associated with implant survival rates. However, the quality of the bone obtained after the application of certain grafting materials has not been fully elucidated yet. The aims of this multicenter study were to analyse histomorphometrical samples obtained 6 months after sinus grafting using a composite graft consisting of anorganic bovine bone (ABB) + autologous bone (AB), and to compare these samples with maxillary pristine bone biopsies.

Material and methods: Ninety maxillary sinus augmentations were performed for delayed implant placement $(N=90)$ in 45 consecutive patients (test group). Bone cores were harvested 6 months after grafting for histomorphometric and ultrastructural study. Control pristine bone biopsies were taken from the posterior maxilla of 10 patients (control). Bone radiographic changes were assessed up to 24 months after implant loading.

Results: The total mean values after analysis of test cores revealed a proportion of $46.08 \pm 16.6 \%$ of vital bone, $42.27 \pm 15.1 \%$ of non-mineralized connective tissue, and $37.02 \pm 25.1 \%$ of the remaining ABB particles. Significant bone remodeling activities were noticed in sinus grafting samples when compared with pristine bone. A statistically significant difference was observed in the number of osteoid lines between two groups, with higher values in the test one $(15.1 \pm 11.48 \%$ vs. $2.5 \pm 2.2 \%, P=0.0005)$.

Ultrastructural study showed that vital trabecular bone was in intimal contact with $A B B$ particles. Radiographic analysis revealed that the higher the proportion of remaining $A B B$, the lower the total vertical resorption of the graft.

Conclusion: Sinus grafting constitutes an excellent model for the study of de novo bone formation patterns and graft consolidation, when a combination of different bone substitutes is applied. The combination of $A B B+A B$ yields highly satisfactory outcomes from both a clinical and a histologic perspective.
\end{abstract}

Bone availability is the key for successful implant placement (Block \& Kent I997). Implant primary stability is often challenging in the posterior segments of the maxilla. This is because of the poor osseous density typically found in this region, insufficient bone availability mainly due to sinus pneumatization, and alveolar bone atrophy as a consequence of tooth loss (Yildirim et al. 2000). Although the use of short or tilted implants has been shown to be a suitable therapeutic option to overcome these obstacles (Aparicio et al. 200I; Morand \& Irinakis 2007), in cases of 
anatomical limitations and consideration of prosthesis long-term biomechanical stability, sinus grafting has emerged as a good option for implant site development to facilitate implant placement.

Since the early introduction of sinus grafting in implant dentistry (Boyne \& James 1980), several modifications of this technique have been proposed in the literature throughout the last two decades $(\mathrm{Ta}-$ tum 1986; Summers 1998). Bone grafting material selection is one of the differential factors that may play a significant role in sinus grafting outcomes. The search for an ideal bone grafting material that would enable clinicians to obtain the best mature bone formation in the shortest period of time, with minimal complications, is one of the most investigated topics in this field. Anorganic bovine bone (ABB) is a biocompatible material that presents similar structure and physical properties as human cancellous bone. When ABB combines with autogenous bone, it may represent an excellent grafting material for maxillary sinus augmentation (Wallace \& Froum 2003). Autologous bone (AB) provides cellularity and growth factors while $\mathrm{ABB}$ facilitates space maintenance and slows down the process of rapid bone remodeling of $\mathrm{AB}$. This may be explained by $\mathrm{ABB}^{\prime} \mathrm{s}$ slow resorption rate and its osteoconductive properties (Schlegel et al. 2003).

A modified sinus grafting technique using a bone scraper to simultaneously access the Schneiderian's membrane and collect a part of the bone that lines the lateral wall of the sinus cavity, to be later used as a grafting material in combination with $\mathrm{ABB}$, has been previously proposed by our group (Galindo-Moreno et al. 2007). Several authors have reported excellent clinical outcomes after using a combination of $\mathrm{ABB}$ and $\mathrm{AB}$ for sinus grafting (Yildirim et al. 200I; Hallman et al. 2002; GalindoMoreno et al. 2008). In spite of this, little information is available regarding the biologic response after performing sinus grafting using a combination of xenogenic material $(A B B)$ and autogenous bone $(A B)$. Therefore, the main aim of the present study was to analyse, morphologically and histomorphometrically, samples obtained 6 months after sinus grafting of grafted sinuses of human subjects using a composite graft consisting of $\mathrm{ABB}+\mathrm{AB}$, and to find out the differences with maxillary pristine bone.

\section{Material and methods}

\section{Study design}

In this multicenter, prospective, controlled clinical study, 45 patients $(28$ males and $\mathrm{I} 7$ females) in need of a bilateral sinus grafting procedure to allow replacement of missing posterior maxillary teeth with implantsupported restorations were recruited for the study after informed consent. Information regarding medical and dental history was recorded following a questionnaire. To be included in the study, patients had to be adults between 18 and 85 years of age, present a physical status according to the American Society of Anesthesiologists (ASA) of I or II, have neither an uncontrolled systemic disease nor a condition known to alter bone metabolism (i.e. osteoporosis, diabetes mellitus ..., etc), adequate oral hygiene $\left(\mathrm{O}^{\prime}\right.$ Leary plaque score $\leq 20 \%)$, and have $<5 \mathrm{~mm}$ bone height remaining after radiographic measurement over panoramic films (Zinner \& Small 2004). Subjects who had taken antibiotics in the previous 3 months, or reported longterm use (>6 months) of medications known to modify bone metabolism (such as bisphosphonates, corticosteroids ..., etc), women who were pregnant or attempting to get pregnant at the time of the screening, and patients who presented existing sinus conditions, sepsis, a history of cancer and/or radiation to the oral cavity, or complications derived from any of these conditions affecting the sinus area were excluded from the study.

A total of 90 grafting procedures were conducted. Patients underwent a delayed implant placement approach. Additionally, Io subjects (five males and five females) consented in order to harvest control biopsies. Ten pristine bone core biopsies were harvested from the posterior maxilla at the time of implant placement from sites where bone grafting was not necessary.

This study was conducted according to the principles of WHO Declaration of Helsinki (Schuklenk \& Ashcroft 2000).

\section{Surgical and restorative procedure}

All patients took 875/I25 mg of amoxicillin/clavulanic acid, every $8 \mathrm{~h}$ starting I day before the surgery. Clindamycin, $300 \mathrm{mg}$ every $8 \mathrm{~h}$, was prescribed to penicillinallergic patients. This medication was maintained for 7 additional days. All sur- gical procedures were performed under local anesthesia (Ultracain ${ }^{\circledR}$, Aventis Inc., Frankfurt, Germany). A modification of the conventional lateral wall approach was used to perform the sinus grafting in all patients. A bone scraper (Safe scraper ${ }^{\mathrm{B}}$, Meta corp., Remigia, Italy) was used to harvest autologous cortical bone and to expose the Schneiderian membrane. After membrane elevation, all sinus cavities were grafted with $\mathrm{AB}$ in combination $\mathrm{ABB}$ (Bio-Oss $^{B}$ particle size $250-1000 \mu \mathrm{m}-$ Geistlich Pharma AG, Wolhusen, Switzerland), mixed in a I : I volume ratio. After bone grafting, an absorbable collagen membrane (Bio-Gide ${ }^{\mathbb{B}}$ - Geistlich Pharma AG) was placed over the window to minimize soft tissue invasion. Primary closure was achieved in all cases, suturing with 3-O silk suture(Laboratorio Aragó, Barcelona, Spain).

After a 6-month healing period, a 3-mm internal diameter, 4-mm external diameter trephine was used to collect bone core biopsies from the sites where implants had to be placed, according to the restorative plan. Hence, the initial osteotomy was performed using a trephine, and not with the conventional drills of each implant system. According to the study protocol, just one bone core per sinus was analyzed for conventional histological and histomorphometrical study. The implant placed in the ideal position of the maxillary first molar was the preferred site for bone sample collection. Other cores were taken from different implant positions for ultrastructural analysis. A total of 90 implants represented the group that was followed up: 52 Microdent ${ }^{\mathrm{B}}$ (Microdent Implant System, Barcelona, Spain) and 38 Astra Tech /Osseospeed ${ }^{\text {TM }}$, Astra Tech, Mölndal, Sweden) with a range I3-16 mm of length and $4.2-5 \mathrm{~mm}$ of width. A staged approach was performed in all cases; therefore, 6 months after implant placement, a second-stage surgery was performed. After soft tissue healing, impressions were taken. The final screw-retained implant-supported PFM crowns were delivered - approximately 2 weeks later - and an occlusal adjustment was performed. After implant loading, all patients were placed in a 3 -month maintenance program.

\section{Radiographic variables}

Standardized digital panoramic films of each patient were obtained before and immediately after sinus grafting surgery, 
before implant insertion, at the time of prosthesis delivery, and 24 months after functional loading (Kodak ACR-2000, Eastman Kodak Company, Rochester, NY, USA). Total bone height, at the point of shortest remaining alveolar bone preoperatively, as well as the maximum vertical augmentation after grafting and on the day of implant placement was measured by the same examiner using a specific software (Digident Dent-A-View Version I.O, DigiDent, DIT, Nesher, Israel).

\section{Histological study}

Bone core biopsies were fixed in Io\% buffered formalin for $24 \mathrm{~h}$. They were subsequently decalcified in a decalcifier containing formaldehyde (Io\% w/v), formic acid $(8 \% \mathrm{w} / \mathrm{v})$, and methanol ( $\mathrm{x} \% \mathrm{w} / \mathrm{v})$ (Surgipath ${ }^{\mathbb{B}}$ Europe Ltd., Peterborough, UK), for at least 8 days, and then embedded in paraffin. Sections were dewaxed, hydrated, and $4 \mu \mathrm{m}$ sections following the central axis of the biopsies were obtained and stained with Hematoxylin-Eosin, periodic acid Schiff's, Masson's trichrome, and Goldner's trichrome techniques. A millimeter scale in the eyepiece of a microscope $\mathrm{BH}_{2}$ (Olympus Optical Company, Ltd., Tokyo, Japan) with a $\times 40$ objective was used to count the osteoblasts, osteoclasts, and osteocytes per $\mathrm{mm}^{2}$. The results were expressed as the number of positive cells per $\mathrm{mm}^{2}$.

Bone histomorphometry was performed semi-automatically on Masson trichromestained sections, assessing Io randomized images with $\times$ Io objective, using a microscope equipped with a digital camera DP70 (Olympus) connected to a computer, and applying Image J software (NIH, USA, http:// rsb.info.nih.gov/ij//. Separate quantifications of vital bone, $\mathrm{ABB}$ particles, and nonmineralized tissue were performed and expressed as percentages of each compartment. The number of osteoid lines on the whole length of the biopsy core was also recorded.

\section{Electron microscopy study}

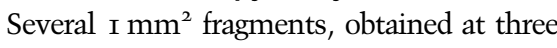
different levels of each sample (coronal, medial, and apical), including trabecular and cortical bone, were fixed in $2.5 \%$ glutaraldehyde solution for $24 \mathrm{~h}$ and subsequently decalcified for 8 days (Decalcifier $\mathrm{I}^{\mathbb{R}}$, Surgipath ${ }^{\mathbb{B}}$ Europe Ltd., Peterborough, UK). Samples were then postfixed in $\mathrm{I} \%$ $\mathrm{OsO}_{4}$ at $4{ }^{\circ} \mathrm{C}$ for $2 \mathrm{~h}$, washed in distilled water, dehydrated in increasing concentrations of acetone, and embedded in Epon. Semithin sections were stained with toluidine blue solution. Ten blocks of LC cluster areas were sampled. Ultrathin (about $70 \mathrm{~nm}$ thick) sections were obtained using an ultramicrotome (Reichert-Jung ULTRACUT E, Leica Microsystems, Barcelona, Spain) and stained with lead citrate and uranyl acetate. Sections were examined with a transmission electron microscope (Zeiss EM 902, Barcelona, Spain) and processed using analySIS ${ }^{B}$ for Windows (Soft Imaging System, Münstes, Germany).

\section{Statistical analysis}

A multilevel model technique with the Box \& Cox transformation was carried out and a Skewnee/Kurtosis test was used to determine the normality of the variables. Because of the high values of intraclass correlation coefficient and non-normal distribution of variables, we decided to use each patient separately as the unit analysis. This was chosen in order to eliminate intrapatient variable high resemblance; hence, we calculated the average value of each variable taken of each patient (considering both sinuses). This required to redefine a new statistical sample where the ' $n$ ' value was 45 , which finally accounted for non-related samples from 45 different patients to determine the statistical significance of the findings. As a consequence, Welch's $t$-test for values with different variance and Pearson's correlation with transformed data were assessed. A $P$ value of $<0.05$ was considered statistically significant. Statistical analysis was performed using specialized software [STATA IO. I program, StataCorp LP, TX, USA, and SPSS-Windows I5.O program, SPSS Inc., Chicago (IL), USA].

\section{Results}

\section{Patients}

Patients' age ranged from 35 to 72 years (mean 50.4 years) in the test group. On the other hand, control patients' ages ranged from 38 to 6I years (mean 48.3 years). Only one perforation of the Schneiderian membrane after elevation and no other complications related to the grafting technique was observed over the study period. At the time of harvesting the bone core biopsy, all cores presented similar dimensions (approximately I $4 \mathrm{~mm}$ ). All patients completed the study.

\section{Radiographic parameters}

The most significant finding regarding radiographic changes was that more bone resorption was observed in patients whose biopsies showed a lower proportion of $\mathrm{ABB}$ particles (<30\% ABB: $0.42 \pm 0.13 \mathrm{~mm}$ vs. $\geq 30 \% \quad$ ABB: $\quad 0.2 \mathrm{I} \pm$ o.I mm, $\quad P=0$ Welch's test), and a higher proportion of non-mineralized tissue (nMT) $(<40 \%$ nMT: $0.22 \pm$ O.II mm vs. $\geq 40 \%$ nMT: $0.39 \pm$ o.I $4 \mathrm{~mm}, P=$ O.OI Welch's test).

\section{Morphologic and histomorphometric analysis}

Analysis of maxillary pristine bone biopsies (Fig. Ia) revealed the presence of mineralized tissue, including mainly trabecular bone, and bone marrow. The mean values after morphometric analysis showed a predominance of bone marrow with adipocytes $(5 \mathrm{I} .2 \pm 8 . \mathrm{I} \%)$, while mineralized tissue was found in a lower $(45.7 \pm 7.9 \%)$, but similar proportion (Table I). A scant number of osteoid lines were observed in association with mineralized structures. The number of osteoblasts, osteoclasts, and osteocytes per squared millimeter is also shown in Table I.

Woven and lamellar type of trabecular bone, in the presence of ABB particles, was observed in samples obtained from patients who underwent sinus augmentation, after 6 months of healing (Fig. Ib). At this time, biopsies from sinus floor elevation showed trabecular bone in a wide range of proportion (from $29.5 \%$ to $75 \%$ ), with normally appearing bone marrow and $\mathrm{ABB}$ particles in and between the trabecular bone, with an appearance of a 'terrazzo floor', especially in the apical portion of the sample (Fig. 2).

The total mean values after image analysis revealed a proportion of $46 . \mathrm{I} \pm \mathrm{I} 6.6 \%$ of vital bone, $42.3 \pm$ I $5.1 \%$ of non-mineralized tissue, and $37 \pm 25 . \mathrm{I} \%$ of remaining ABB particles. Significant bone remodeling activities were noticed in sinus grafting samples as compared with pristine bone. For example, a statistically significant difference in the number of osteoid lines was observed between the two groups, with higher values in the test group (I5.I \pm I I. $48 \%$ vs. $2.5 \pm 2.2 \%, P=0.0005$ ) (Table I). No acute or chronic inflammatory cell 
infiltrate or foreign body reactions were observed in any sample, other than a tendency from the osteoclasts to show activity around ABB particles (Fig. 3). Ultrastructural study showed that vital trabecular bone was in intimal contact with $\mathrm{ABB}$ particles. Also, cement lines between vital bone and $\mathrm{ABB}$ were observed (Fig. 4a), a finding that was previously observed under light microscopy as well (Fig. 4b).

Non-mineralized tissue appeared to be directly correlated with osteoblast number

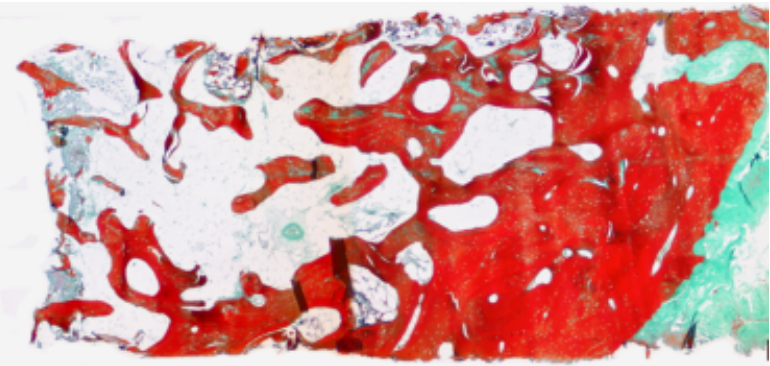

b

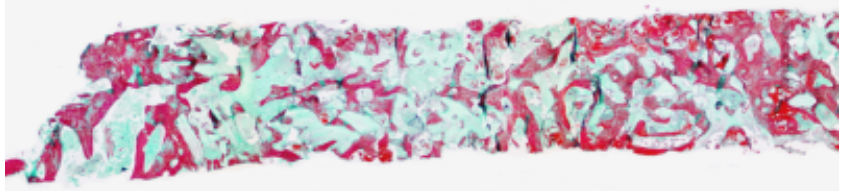

per $\mathrm{mm}^{2}(\rho=0.329, P=0.043)$. In fact, stratified data analysis of samples depending on the proportion of non-mineralized tissue showed that there was a significant difference in osteoblasts $/ \mathrm{mm}^{2}$ when the proportion of interposed soft tissue was higher than $40 \%(\geq 40 \%$ mean $344.9 \pm$ 284 vs. $\quad<40 \% \quad 88.7 \pm 59, \quad P=0.0008$ Welch's test), but the number of osteoid lines was not significant (2I.25 \pm 8.64 vs. I $2.42 \pm$ I $1.68, P=0.99$ Welch's test). However, the remaining ABB particle proportion seemed to be important for osteoid formation $(r=0.509, P=0.00 \mathrm{I})$, as a significant increase of osteoid lines was observed when $\mathrm{ABB}$ proportion was higher than $30 \%$ (I $9.32 \pm 9.01$ vs. I $2.69 \pm$ I2.22, $P=0.012$, Welch's test). Table 2 shows the Pearson's correlation coefficients between morphometric variables.

\section{Discussion}

Primary stability is one of the key factors for successful osseointegration. It is widely

Fig. I. (a) Bone core biopsy obtained from the pristine bone group that showed cortical and trabecular bone formation (Masson trichrome $\times 4$ - scale bar I $\mathrm{mm}$ ). (b) Bone core biopsy from the test group (sinus grafting using autologous and xenogenous bone) (Masson trichrome $\times 2-$ scale bar I $\mathrm{mm}$ ).

Table 1. Morphometric comparison between pristine maxillary bone and grafted bone core biopsies after sinus augmentation

\begin{tabular}{lccc} 
Variables & Pristine bone $(N=10)$ & Grafted bone $(N=45)$ & $P$-values $($ Welch's test) \\
\hline Osteoblasts $\left(\mathrm{no} . / \mathrm{mm}^{2}\right)$ & $247.31 \pm 251.6^{*}$ & $267.26 \pm 266.3$ & 0.92 \\
Osteoclasts $\left(\mathrm{no} . / \mathrm{mm}^{2}\right)$ & $48.38 \pm 72.13$ & $151.1 \pm 192.74$ & 0.51 \\
Osteocytes $\left(\mathrm{no} . \mathrm{mm}^{2}\right)$ & $1575.28 \pm 196.94$ & $1042.66 \pm 807.96$ & 0.0006 \\
Osteoid lines (no.) & $2.5 \pm 2.17$ & $15.1 \pm 11.48$ & 0.0005 \\
Vital bone (\%) & $45.73 \pm 7.98$ & $46.08 \pm 16.63$ & 0.81 \\
Anorganic bovine bone (\%) & $-37.02 \pm 25.09$ & - \\
Non-mineralized connective tissue (\%) & $51.23 \pm 8.1$ & $42.27 \pm 15.1$ & 0.12 \\
\hline
\end{tabular}

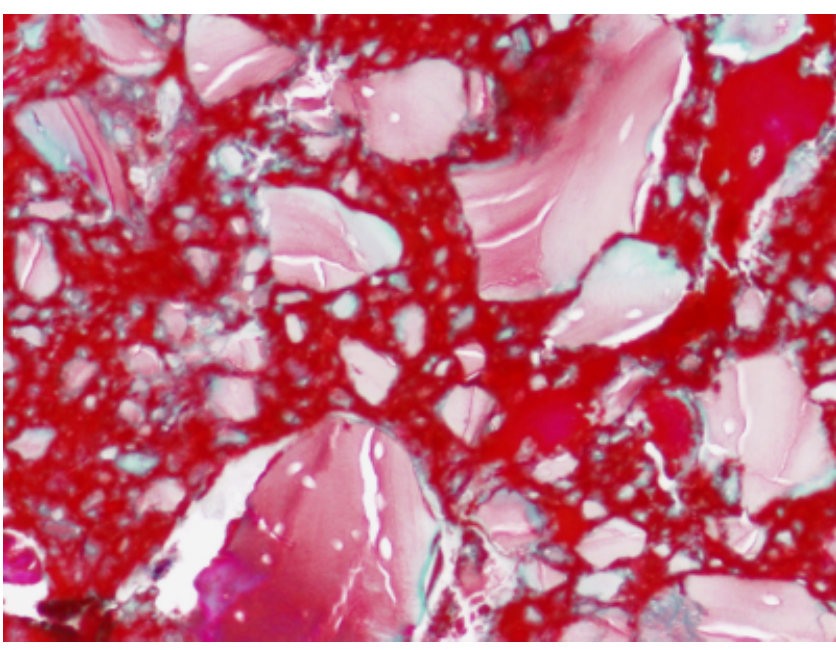

Fig. 2. Bone core biopsy from the test group (sinus grafting using autologous and xenogenous bone) showing a 'terrazzo-floor' image due to microparticulation of anorganic bovine bone (Masson trichrome $\times 20$ ).

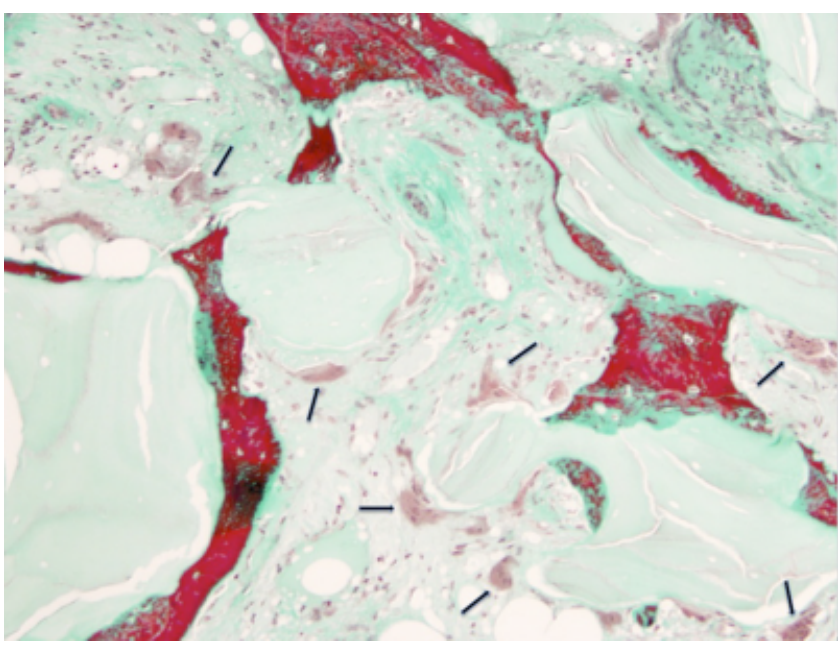

Fig. 3. Bone core biopsy from the test group with numerous osteoclasts (arrows) around the anorganic bovine bone particles (Masson trichrome $\times 20)$. 
acknowledged that primary stability is highly associated with the proportion of mineralized tissue present in a particular location (Turkyilmaz et al. 2007; Turkyilmaz 2008). Therefore, a lack of bone height in the posterior maxilla often requires bone augmentation before implant placement. Sinus grafting is aimed at increasing the amount of available bone in the posterior segments of the maxilla, thus providing an adequate condition for osseointegration after implant placement. The aims of the present study were to evaluate the bone maturation status and histomorphometric features of samples obtained 6 months after sinus grafting, and to compare these parameters with a
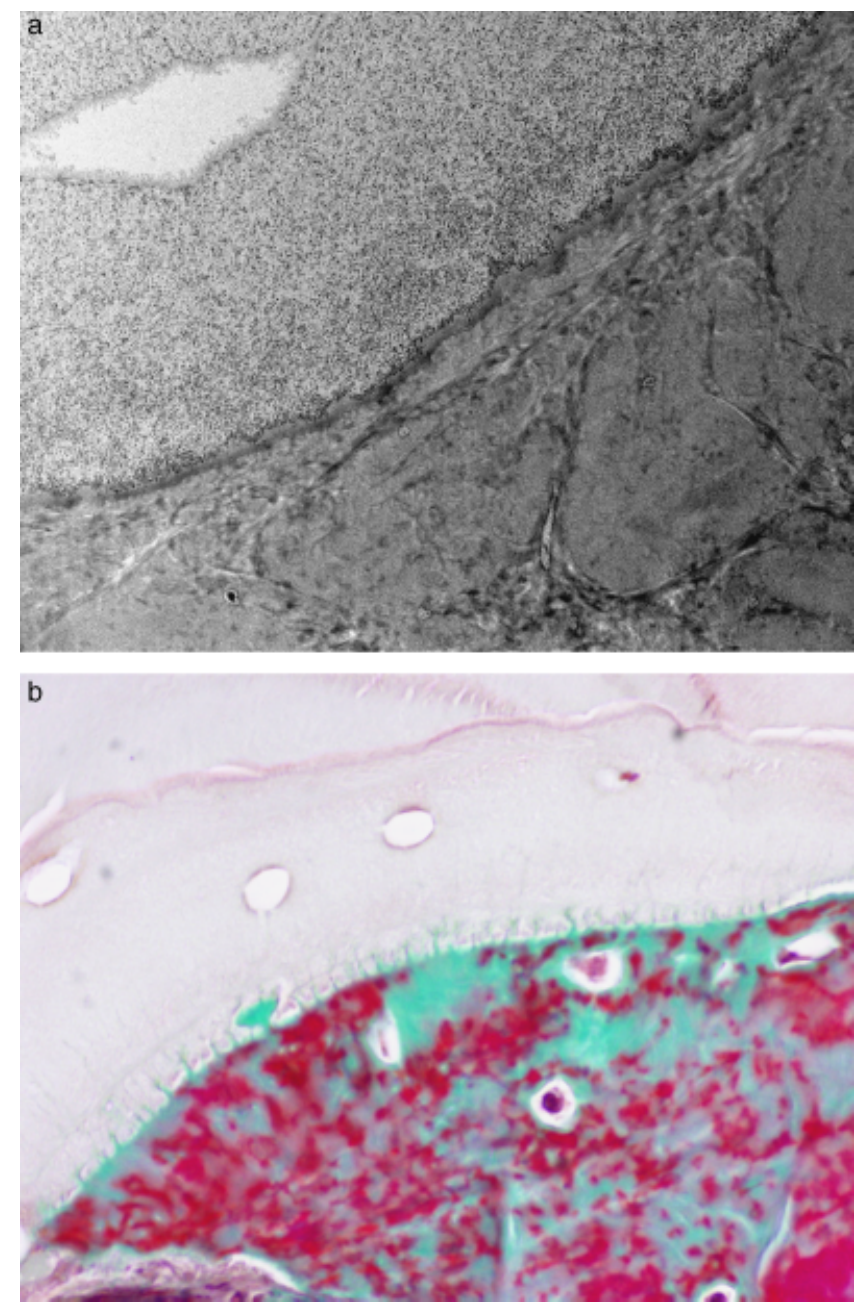

Fig. 4. A narrow relationship between trabecular bone and $\mathrm{ABB}$ particles in the absence of gaps or fibrous interface: (a) ultrastructural image of a cement line between ABB particles and decalcified vital bone (TEM $\times 4600$ ), (b) light microscopy (Masson trichrome $\times$ roo).

pristine bone control. Mineralized vital bone proportion in maxillary pristine bone varies depending on the age and gender, ranging from $23 \%$ to $28 \%$ as shown by previous studies (Trisi \& Rao I999; Ulm et al. I999). In the present study, the mean proportion of mineralized vital bone was found to be $45.73 \pm 7.98 \%$, which is significantly higher than the values reported by other authors. This difference may be explained by the low number of subjects analysed and the particular characteristics of our population. A high percentage of vital bone was noted in pristine bone sections, but it had less signs of bone remodeling when compared with sinus grafting samples.

Sinus grafting success may be evaluated by performing a histologic analysis to determine the features and the amount of newly formed bone that could potentially be in intimal contact with an implant fixture placed in the grafted area (Yildirim et al. 2000). Thus, it has been proposed that maximum osteogenesis of qualitatively high-grade living bone can be used as a way to assess the success outcome of sinus augmentation (Wallace et al. I996). In a previous pilot study, our group found that the mean value for mineralized vital bone was approximately 31\% (GalindoMoreno et al. 2008). This percentage is in accordance with the results previously reported, which found a similar \% of vital bone (ranged from $22.2 \%$ to $34.2 \%$ ) in their samples (Yildirim et al. 2000; Froum et al. 2008; Simunek et al. 2008). In contrast, the mean percentage of vital bone obtained in the present series is one of the highest reported so far for this particular combination of biomaterials $(46.08 \%)$, closely followed by the results reported by Cordaro and colleagues $(45.2 \%)$ and by Artzi and colleagues (4I.6\% after 9 months) (Artzi et al. 2008;

Table 2. Statistical correlations between morphometric variables in grafted bone core biopsies after sinus augmentation

\begin{tabular}{|c|c|c|c|c|c|c|c|}
\hline & $\begin{array}{l}\text { Osteoblasts } \\
\left(\mathrm{mm}^{2}\right)\end{array}$ & $\begin{array}{l}\text { Osteoclasts } \\
\left(\mathrm{mm}^{2}\right)\end{array}$ & $\begin{array}{l}\text { Osteocytes } \\
\left(\mathrm{mm}^{2}\right)\end{array}$ & $\begin{array}{l}\text { Vital bone } \\
(\%)\end{array}$ & $\begin{array}{l}\text { Osteoid } \\
\text { lines (no.) }\end{array}$ & $\begin{array}{l}\text { ABB particles } \\
(\%)\end{array}$ & $\begin{array}{l}\text { Connective } \\
\text { Tissue (\%) }\end{array}$ \\
\hline Osteoblasts $\left(\mathrm{mm}^{2}\right)$ & $1^{*}$ & & & & & & \\
\hline Osteoclasts $\left(\mathrm{mm}^{2}\right)$ & $0.345(0.033)$ & 1 & & & & & \\
\hline Vital bone (\%) & $0.454(0.004)$ & $0.325(0.046)$ & $0.734(0)$ & 1 & & & \\
\hline Osteoid lines (no.) & $0.35(0.03)$ & $0.562(0)$ & $0.544(0)$ & $0.628(0)$ & 1 & & \\
\hline ABB particles (\%) & $-0.247(0.133)$ & $0.296(0.071)$ & $-0.243(0.141)$ & $-0.192(0.247)$ & $0.509(0.001)$ & 1 & \\
\hline
\end{tabular}


Cordaro et al. 2008). This may explain the previously reported higher success rates of implants inserted into grafted sinuses $(\mathrm{Ol}-$ son et al. 2000; Scarano et al. 2006).

Our group has advocated for a surgical approach in which cortical $\mathrm{AB}$ is obtained from the lateral wall of the sinus cavity using a bone scraper, and then combined with a xenogenic bone substitute (e.g., ABB) (Galindo-Moreno et al. 2007; Martos Diaz et al. 2007). This approach not only allows to harvest $\mathrm{AB}$ to be used as a part of the grafting material, but also eliminates the need for a second surgical site to harvest autogenous bone. Despite $\mathrm{AB}$ being regarded as the gold standard in bone grafting material, it undergoes a rapid resorption when compared with other grafting materials. This resorption can be as much as $60 \%$ when it is used as a single biomaterial (Jensen \& Sindet-Pedersen I99I). In this sense, Schlegel and collaborators observed that $\mathrm{AB}$ may be resorbed up to $40 \%$ after 6 months of healing when used as a sinus grafting material. They also found a lower bone-to-implant contact for implants placed in areas where $\mathrm{AB}$ was used vs. ABB: $42 \%$ vs. $68 \%$, respectively (Schlegel et al. 2003). Hence, the combination of xenogeneic and autologous bone may provide some benefits when compared with the sole use of $\mathrm{AB}$. These include unlimited $\mathrm{ABB}$ availability, allowing recolonization and apposition of osteogenic cells, and a slow resorption rate (Piattelli et al. I999; Taylor et al. 2002). Although it has not been fully elucidated what is the average amount of resorption in a determined time span, it has been shown that ABB particles may be present even 9 years after grafting (Traini et al. 2007). Our results showed a mean percentage of remaining $\mathrm{ABB}$ particle of $37.01 \%$ after 6 months of healing. This supports the notion of a slow resorption rate. $\mathrm{ABB}$ is an adequate scaffold for dimensional graft maintenance, a concept that is reinforced by the findings of this study, because the higher the proportion of $\mathrm{ABB}$, the lower the total mean resorption of the graft. Stratified data analysis of $\mathrm{ABB}$ percentage present in our samples showed that, above $30 \%$ of the remaining xenogeneic particle, graft resorption was significantly lower (0.2 I \pm O.I mm vs. $0.42 \pm$ O.I3 mm, $P=$ o Welch's test). We assume that a slow degradation of the material occurs and not a complete replacement by $\mathrm{AB}$ in $<5$ months after grafting, as reported by Tadjoedin et al. (2003). In addition, the composite grafting material proposed here promotes an early osteogenesis/osteoinduction elicited by the cells and growth factors of the $\mathrm{AB}$, which may in turn allow for a more efficient implant osseointegration (Tadjoedin et al. 2003).

Newly formed bone showed slightly more osteoblasts (mean $\left.\# / \mathrm{mm}^{2}=267.3\right)$, more osteoid lines $(P<0.46$ Welch's test $)$, significantly more osteoclasts (mean \#/ $\left.\mathrm{mm}^{2}=\mathrm{I} 5 \mathrm{I} . \mathrm{I}\right)$, and a higher proportion of osteocytes (four times higher than osteoblasts), but less than mature pristine bone $(P<0.0006$, Welch's test $)$. A direct, statistically significant correlation found between the number of osteoblasts and the quantity of non-mineralized tissue supports the observations of Zerbo et al. (2005), who showed that early differentiation towards preosteoblastic undifferentiated cells may be mediated by the expression of Run $\times 2$, sialoprotein, and osteopontin.

On the other hand, the remaining $\mathrm{ABB}$ particle proportion seemed to be important for osteoid formation $(r=0.509, P=0.00 \mathrm{I})$, as a significant increase of osteoid lines was observed when the $\mathrm{ABB}$ proportion was $>30 \%$ (I9.32 \pm 9.01 vs. I $2.69 \pm$ I 2.22 , $P=$ o.or 2, Welch's test). This finding may be explained by the osteoconductive nature of ABB (Schlegel et al. 2003). Ultrastructural analysis revealed that the remaining ABB particles were surrounded by newly formed mature bone, throughout all the samples. Cement lines could be clearly identified, in the absence of gaps or fibrous interfaces. In the light of the findings of this study, we can state that $\mathrm{ABB}$ shows a different behavior from other bone substi- tutes, such as tricalcium phosphate (Wiltfang et al. 2003; Zerbo et al. 2004), being attributable to its high osteoconductivity. However, we should not forget that, when no perforation is present, the sinus cavity is a self-contained defect. This promotes space maintenance and graft stability, and allows cell migration and continuous blood supply from all the bony walls, which results in favorable healing conditions.

\section{Conclusions}

We consider that sinus grafting constitutes an excellent model for the study of de novo bone formation patterns and graft consolidation when a combination of different bone substitutes is applied. In this study, we propose the use of a composite graft, consisting of $\mathrm{AB}$ and $\mathrm{ABB}$. The results obtained are highly satisfactory from both a clinical and a histologic perspective. However, many questions remain unanswered with regard to this exciting field. For instance, why the non-mineralized tissue present in our samples determines the newly formed vital bone cellularity and how the quantity of $\mathrm{ABB}$ prevents the resorption.

Acknowledgements: We would like to acknowledge the contribution of María Dolores Rodríguez, Jorge A. Payá, and Mohamed Tassi, technicians at the Department of Pathology and IBIMER, as well as Dr Luna for his special contribution in the statistical analysis. This research project was partially supported by research group grants \#CTS-138 and \#CTS 583 (Junta de Andalucía, Spain).

Disclosure: All the authors claim to have no financial interest, either directly or indirectly, in any company or any of the products mentioned in this manuscript. Disclaimers: The authors do not have any financial interests, either directly or indirectly, in the products listed in the study.

\section{References}

Aparicio, C., Perales, P. \& Rangert, B. (200I) Tilted implants as an alternative to maxillary sinus grafting: a clinical, radiologic, and periotest study. Clinical Implant e) Dental Related Research 3 39-49.
Artzi, Z., Weinreb, M., Carmeli, G., Lev-Dor, R., Dard, M. \& Nemcovsky, C.E. (2008) Histomorphometric assessment of bone formation in sinus augmentation utilizing a combination of autogenous and hydroxyapatite/biphasic tricalcium phosphate graft materials: at 6 and 9 months in humans. Clinical Oral Implants Research I9: 686-692.

Block, M.S. \& Kent, J.N. (I997) Sinus augmentation for dental implants: the use of autogenous 
bone. Journal of Oral and Maxillofacial Surgery 55: I28I-I 286.

Boyne, P.J. \& James, R.A. (I980) Grafting of the maxillary sinus floor with autogenous marrow and bone. Journal of Oral Surgery 38: 6I 3-6I 6.

Cordaro, L., Bosshardt, D.D., Palattella, P., Rao, W., Serino, G. \& Chiapasco, M. (2008) Maxillary sinus grafting with bio-oss or straumann bone ceramic: histomorphometric results from a randomized controlled multicenter clinical trial. Clinical Oral Implants Research r9: 796-803.

Froum, S.J., Wallace, S.S., Cho, S.C., Elian, N. \& Tarnow, D.P. (2008) Histomorphometric comparison of a biphasic bone ceramic to anorganic bovine bone for sinus augmentation: 6- to 8month postsurgical assessment of vital bone formation. A pilot study. International Journal of Periodontics and Restorative Dentistry 28: 273$28 \mathrm{I}$.

Galindo-Moreno, P., Avila, G., Fernandez-Barbero, J.E., Aguilar, M., Sanchez-Fernandez, E., Cutando, A. \& Wang, H.L. (2007) Evaluation of sinus floor elevation using a composite bone graft mixture. Clinical Oral Implants Research I8: 376-382.

Galindo-Moreno, P., Avila, G., Fernandez-Barbero, J.E., Mesa, F., O'Valle-Ravassa, F. \& Wang, H.L. (2008) Clinical and histologic comparison of two different composite grafts for sinus augmentation: a pilot clinical trial. Clinical Oral Implants Research I9: 755-759.

Hallman, M., Sennerby, L. \& Lundgren, S. (2002) A clinical and histologic evaluation of implant integration in the posterior maxilla after sinus floor augmentation with autogenous bone, bovine hydroxyapatite, or a 20:80 mixture. The International Journal of Oral $\oplus$ Maxillofacial Implants I7: 635-643.

Jensen, J. \& Sindet-Pedersen, S. (I99I) Autogenous mandibular bone grafts and osseointegrated implants for reconstruction of the severely atrophied maxilla: a preliminary report. Journal of Oral and Maxillofacial Surgery 49: I277-I 287.

Martos Diaz, P., Naval Gias, L., Sastre Perez, J., Gonzalez Garcia, R., Bances del Castillo, F., Mancha de la Plata, M., Galindo Moreno, P. \& Munoz Guerra, M. (2007) Sinus elevation by in situ utilization of bone scrapers: technique and results. Medicina Oral Patologia Oral y Cirugia Bucal I2: E537-E54I.

Morand, M. \& Irinakis, T. (2007) The challenge of implant therapy in the posterior maxilla: providing a rationale for the use of short implants. Journal of Oral Implantology 33: 257-266.

Olson, J.W., Dent, C.D., Morris, H.F. \& Ochi, S. (2000) Long-term assessment (5 to 7I months) of endosseous dental implants placed in the augmen- ted maxillary sinus. Annals of Periodontology 5 : I $52-156$.

Piattelli, M., Favero, G.A., Scarano, A., Orsini, G. \& Piattelli, A. (I999) Bone reactions to anorganic bovine bone (bio-oss) used in sinus augmentation procedures: a histologic long-term report of 20 cases in humans. The International Journal of Oral et Maxillofacial Implants I4: 835-840.

Scarano, A., Degidi, M., Iezzi, G., Pecora, G., Piattelli, M., Orsini, G., Caputi, S., Perrotti, V., Mangano, C. \& Piattelli, A. (2006) Maxillary sinus augmentation with different biomaterials: a comparative histologic and histomorphometric study in man. Implant Dentistry I 5: 197-207.

Schlegel, K.A., Fichtner, G., Schultze-Mosgau, S. \& Wiltfang, J. (2003) Histologic findings in sinus augmentation with autogenous bone chips versus a bovine bone substitute. The International Journal of Oral ↔) Maxillofacial Implants I8: 53-58.

Schuklenk, U. \& Ashcroft, R. (2000) International research ethics. Bioethics I4: I 58-I72.

Simunek, A., Kopecka, D., Somanathan, R.V., Pilathadka, S. \& Brazda, T. (2008) Deproteinized bovine bone versus beta-tricalcium phosphate in sinus augmentation surgery: a comparative histologic and histomorphometric study. The International Journal of Oral e) Maxillofacial Implants 23: 935-942.

Summers, R.B. (I998) Sinus floor elevation with osteotomes. Journal of Esthetic Dentistry Io: I64-I7I.

Tadjoedin, E.S., de Lange, G.L., Bronckers, A.L., Lyaruu, D.M. \& Burger, E.H. (2003) Deproteinized cancellous bovine bone (bio-oss) as bone substitute for sinus floor elevation. A retrospective, histomorphometrical study of five cases. Journal of Clinical Periodontology 30: 26 I-270.

Tatum, H. Jr (I986) Maxillary and sinus implant reconstructions. Dental Clinics of North America 30: $207-229$.

Taylor, J.C., Cuff, S.E., Leger, J.P., Morra, A. \& Anderson, G.I. (2002) In vitro osteoclast resorption of bone substitute biomaterials used for implant site augmentation: a pilot study. The International Journal of Oral $\oplus$ Maxillofacial Implants 17: $32 \mathrm{I}-330$.

Traini, T., Valentini, P., Iezzi, G. \& Piattelli, A. (2007) A histologic and histomorphometric evaluation of anorganic bovine bone retrieved 9 years after a sinus augmentation procedure. Journal of Periodontology 78: 955-961.

Trisi, P. \& Rao, W. (I999) Bone classification: clinical-histomorphometric comparison. Clinical Oral Implants Research Iо: I-7.

Turkyilmaz, I. (2008) Influence of bone density on implant stability parameters and implant success: a retrospective clinical study. BMC Oral Health $\mathbf{8}$ : 32.

Turkyilmaz, I., Tumer, C., Ozbek, E.N. \& Tozum, T.F. (2007) Relations between the bone density values from computerized tomography, and implant stability parameters: a clinical study of 230 regular platform implants. Journal of Clinical Periodontology 34: 716-722.

Ulm, C., Kneissel, M., Schedle, A., Solar, P., Matejka, M., Schneider, B. \& Donath, K. (I999) Characteristic features of trabecular bone in edentulous maxillae. Clinical Oral Implants Research Io: $459-467$.

Wallace, S.S., Froum, S.J. \& Tarnow, D.P. (1996) Histologic evaluation of a sinus elevation procedure: a clinical report. International Journal of Periodontics and Restorative Dentistry I6: 46-5 I.

Wallace, S.S. \& Froum, S.J. (2003) Effect of maxillary sinus augmentation on the survival of endosseous dental implants. A systematic review. Annals of Periodontology 8: 328-343.

Wiltfang, J., Schlegel, K.A., Schultze-Mosgau, S., Nkenke, E., Zimmermann, R. \& Kessler, P. (2003) Sinus floor augmentation with beta-tricalciumphosphate (beta-tcp): does platelet-rich plasma promote its osseous integration and degradation? Clinical Oral Implants Research I4: 2I3-2I8.

Yildirim, M., Spiekermann, H., Biesterfeld, S. \& Edelhoff, D. (2000) Maxillary sinus augmentation using xenogenic bone substitute material bio-oss in combination with venous blood. A histologic and histomorphometric study in humans. Clinical Oral Implants Research II: 217-229.

Yildirim, M., Spiekermann, H., Handt, S. \& Edelhoff, D. (200I) Maxillary sinus augmentation with the xenograft bio-oss and autogenous intraoral bone for qualitative improvement of the implant site: a histologic and histomorphometric clinical study in humans. The International Journal of Oral e) Maxillofacial Implants I6: 23-33.

Zerbo, I.R., Bronckers, A.L., de Lange, G. \& Burger, E.H. (2005) Localisation of osteogenic and osteoclastic cells in porous beta-tricalcium phosphate particles used for human maxillary sinus floor elevation. Biomaterials 26: I445-I 45 I.

Zerbo, I.R., Zijderveld, S.A., de Boer, A., Bronckers, A.L., de Lange, G., ten Bruggenkate, C.M. \& Burger, E.H. (2004) Histomorphometry of human sinus floor augmentation using a porous betatricalcium phosphate: a prospective study. Clinical Oral Implants Research I5: 724-732.

Zinner, I.D. \& Small, S.A. (2004) Maxillary sinus grafts and prosthetic management. In: Zinner, I.D. \& Panno, V., eds. Implants Dentistry: From Failure to Success. Ist edition, 99-100. Hong Kong: Quintessence Books. 